Original Article

\title{
Percentage of importance indice-production unknown: loss and solution sources identification on system
}

\author{
Percentagem de índice de importância-produção desconhecida: identificação de \\ fontes de perda e de solução no sistema

\section{G. L. Demolin-Leite ${ }^{\mathrm{a} *}$ (1)} \\ aUniversidade Federal de Minas Gerais - UFMG, Instituto de Ciências Agrárias, Insetário G. W. G. Moraes, Montes Claros, MG, Brasil
}

\begin{abstract}
Indices are used to help on decision-making. This study aims to develop and test an index, which can determine the loss (e.g., herbivorous insects) and solution (e.g., natural enemies) sources. They will be classified according to their importance regarding the ability to damage or to reduce the source of damage to the system when the final production is unknown. Acacia auriculiformis (Fabales: Fabaceae), a non-native pioneer species in Brazil with fast growth and rusticity, is used in restoration programs, and it is adequate to evaluate a new index. The formula was: Percentage of the Importance Indice-Production Unknown (\% I.I. $-P U)=\left[\left(k s_{1} x c_{1} x d s_{1}\right) / \Sigma\left(k s_{1} x c_{1} x d s_{1}\right)\right.$ $\left.+\left(k s_{2} \times c_{2} \times d s_{2}\right)+\left(k s_{n} \times c_{n} \times d s_{n}\right)\right] \times 100$. The loss sources Aethalion reticulatum L., 1767 (Hemiptera: Aethalionidae), Aleyrodidae (Hemiptera), Stereoma anchoralis Lacordaire, 1848 (Coleoptera: Chrysomelidae), and Tettigoniidae, and solution sources Uspachus sp. (Araneae: Salticidae), Salticidae (Araneae), and Pseudomyrmex termitarius (Smith, 1877) (Hymenoptera: Formicidae) showed the highest \% I.I.-PU on leaves of A. auriculiformis saplings. The number of Diabrotica speciosa Germar, 1824 (Coleoptera: Chrysomelidae) was reduced per number of Salticidae; that of A. reticulatum that of Uspachus sp.; and that of Cephalocoema sp. (Orthoptera: Proscopiidae) that of $P$. termitarius on A. auriculiformis saplings. However, the number of Aleyrodidae was increased per number of Cephalotes sp. (Hymenoptera: Formicidae) and that of $A$. reticulatum that of Brachymyrmex sp. (Hymenoptera: Formicidae) on A. auriculiformis saplings. The A. reticulatum damage was reduced per number of Uspachus sp., but the Aleyrodidae damage was increased per number of Cephalotes sp., totaling $23.81 \%$ of increase by insect damages on A. auriculiformis saplings. Here I show and test the \% I.I.-PU. It is an new index that can detect the loss or solution sources on a system when production is unknown. It can be applied in some knowledge areas.
\end{abstract}

Keywords: abundance, aggregation, agriculture, chi-squared test, constancy, forestry production, frequency, natural system.

\begin{abstract}
Resumo
Índices são usados para ajudar na tomada de decisões. Este trabalho teve como objetivo desenvolver e testar um índice capaz de determinar fontes de perda (ex.: insetos herbívoros) e de solução (ex.: inimigos naturais). Eles serão classificados de acordo com sua importância quanto a habilidade de danificar ou reduzir danos no sistema, quando a produção final é desconhecida. Acacia auriculiformis (Fabales: Fabaceae), uma espécie pioneira não nativa do Brasil com rápido crescimento e rusticidade, usada em programas de restauração, é adequada para avaliar um novo índice. A fórmula foi: Porcentagem de Índice de Importância-Produção Desconhecida (\% I.I. $-P D)=\left[\left(k s_{1} \times c_{1} \times d s_{1}\right) / \Sigma\right.$ $\left.\left(k s_{1} \times c_{1} \times d s_{1}\right)+\left(k s_{2} \times c_{2} \times d s_{2}\right)+\left(k s_{n} \times c_{n} \times d s_{n}\right)\right] \times 100$. As fontes de perda Aethalion reticulatum L., 1767 (Hemiptera: Aethalionidae), Aleyrodidae (Hemiptera), Stereoma anchoralis Lacordaire, 1848 (Coleoptera: Chrysomelidae) e Tettigoniidae, e as fontes de solução Uspachus sp. (Araneae: Salticidae), Salticidae (Araneae) e Pseudomyrmex termitarius (Smith, 1877) (Hymenoptera: Formicidae) apresentaram maiores \% I.I.-PD nas folhas das mudas de A. auriculiformis. O número de Diabrotica speciosa Germar, 1824 (Coleoptera: Chrysomelidae) foi reduzido pelo número de Salticidae; o de A. reticulatum pelo de Uspachus sp.; e o de Cephalocoema sp. (Orthoptera: Proscopiidae) pelo de P. termitarius em mudas de A. auriculiformis. Entretanto, o número de Aleyrodidae foi aumentado pelo número de Cephalotes sp. (Hymenoptera: Formicidae) e o de A. reticulatum pelo de Brachymyrmex sp. (Hymenoptera: Formicidae) em mudas de A. auriculiformis. O dano de A. reticulatum foi reduzido pelo número de Uspachus sp., mas o dano de Aleyrodidae foi aumentado pelo número de Cephalotes sp., totalizando 23,81\% de aumento de danos em mudas de A. auriculiformis. Aqui eu apresento e testo o \% I.I.-PD. Ele é um novo índice capaz de detectar fontes de perda e de solução no sistema quando não se conhece a produção final. Ele pode ser aplicado em algumas áreas do conhecimento.
\end{abstract}

Palavras-chave: abundância, agregação, agricultura, constância, frequência, produção florestal, sistema natural, teste do chi-quadrado.

*e-mail: germano.demolin@gmail.com

Received: June 12, 2021 - Accepted: October 8, 2021 


\section{Introduction}

Indices are used to help on decision-making, and, whenever possible, determining key factor. They might be crucial in various areas, such as agrarian (Peterson, et al. 2009; Da Silva et al., 2017; Demolin-Leite, 2021), educational (Davis and Wigelsworth, 2018), industrial (Lin et al., 2007), medical (Liu et al., 2017; Goldenberg and Grantcharov, 2019), among others. These indices, in general, use abundance, constancy, and/or frequency and other factors, related to the events. Those can be analyzed by correlation, factor analysis, frequency distribution, matrices, mean or t-test, multiple or simple regression analysis, etc. (Lin et al., 2007; Da Silva et al., 2017; Liu et al., 2017; Goldenberg and Grantcharov, 2019; Demolin-Leite, 2021). Sometimes, indices are complex and laborious to be obtained. The Importance Indice (I.I.) can determine the loss and solution sources on a system in some knowledge areas (e.g., agronomy), since production is known (Demolin-Leite, 2021). Events (eg., agricultural pest) can have different magnitudes (numerical measurements), frequencies, and distributions (aggregate, random, or regular) of event occurrence, and I.I. bases in this triplet (Demolin-Leite, 2021). In general, the higher magnitude and frequency, with aggregated distribution, the greater will be the problem or the solution (eg., natural enemies versus pests) on the system (Demolin-Leite, 2021). However, the final production of the system is not always known or is challenging to measure (e.g., degraded area recovery).

The earleaf acacia, Acacia auriculiformis A. Cunn. ex Benth. (Fabales: Fabaceae), is native from Australia, Papua New Guinea, and Indonesia (Doran and Turnbull, 1997). Its leaves are dense, bipinnate with petioles and size from 8 to $22.5 \mathrm{~cm}$ and 10 to $52 \mathrm{~mm}$ with three longitudinal and many secondary ribs (Doran and Turnbull, 1997). This plant is a priority species for the International Union of Forestry Research Organisations (IUFRO) for research and development in tropical areas (Wickneswari and Norwati, 1993). Its wood is of high quality for particleboard, pulpwood, tannin, and timber (Firmansyah et al., 2020). Acacia spp. (Fabales: Fabaceae) are used to recover degraded areas (Balieiro et al., 2017), although the introduction of non-native plants may impact natural ecosystems. The abiotic characteristics of the area and the life history facilitate the establishment and dispersal of mangium tree, Acacia mangium Willd. (Fabales: Fabaceae), in the Amazonian savannas (Aguiar Junior et al., 2014). On the other hand, the local biotic resistance may reduce the dispersal of introduced Acacia spp. as an invasive species (Londe et al., 2020). The durability of the A. auriculiformis wood is long-term, and the susceptibility to diseases and adaptability to poor soils by this plant is high (Wong et al., 2011; Rahman et al., 2017). Acacia auriculiformis can increase moisture retention, deposition of potassium and organic carbon in the soil (litter). It can also make the phytoextraction of heavy metals from the soil (through mycorrhizal associations) (Rana and Maiti, 2018) and biological fixation of atmospheric nitrogen via bacteria in its roots. Arthropods on this and other Acacia spp. (Rodríguez et al., 2020) have been studied, but their importance is unknown.
The objective of this study was to develop and test an index, which can determine the loss (e.g., herbivores insects) and solution sources (e.g., natural enemies), classifying them according to their importance regarding the ability to damage or reduce the source of damage on 48 A. auriculiformis saplings - system with production unknown.

\section{Material and methods}

\subsection{Experimental site}

This study was carried out in a degraded area ( $\approx 1$ ha) of the "Instituto de Ciências Agrárias da Universidade Federal de Minas Gerais (ICA/UFMG)" in the municipality of Montes Claros, Minas Gerais state, Brazil (latitude 16을 $51^{\prime} 38^{\prime \prime}$ S,

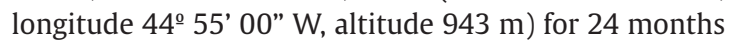
(April 2015 to March 2017). According to the Köppen climate classification, the climate of this area is tropical dry, with annual precipitation and temperature between 1,000 and $1,300 \mathrm{~mm}$ and $\geq 24^{\circ} \mathrm{C}$, respectively (Alvares et al., 2013). The soil is Neosol Litolic with an Alic horizon (Silva et al., 2020).

\subsection{Experimental design}

The A. auriculiformis seedlings were prepared, in March 2014 , in a nursery in plastic bags $(16 \times 24 \mathrm{~cm}$ ) with reactive natural phosphate mixed with the substrate at a dosage of $160 \mathrm{~g}$ and planted, at the same time, in the final site in September of this year. Each A. auriculiformis seedling was planted in a hole $(40 \times 40 \times 40 \mathrm{~cm})$ when they were $30 \mathrm{~cm}$ high with a 2-meter spacing between them. The soil was corrected with dolomitic limestone with the base saturation increased to 50\%, natural phosphate, gypsum, FTE (Fried Trace Elements), potassium chloride, and micronutrients based on the soil analysis. A total of $20 \mathrm{~L}$ of dehydrated sewage sludge with its biochemical characteristics defined (Silva et al., 2020) was placed in a single dose, per hole. The young $48 \mathrm{~A}$. auriculiformis saplings (young trees in the vegetative period) were irrigated twice a week until the beginning of the rainy season (October).

\subsection{Counting the arthropods}

Defoliation - leaf area loss on a $0-100 \%$ scale with $5 \%$ increments for removed leaf area (Silva et al., 2020) - and boring of branches by insects, and score damage by sap-sucking insects: $\mathrm{I}$ = non-damage; $\mathrm{II}=$ appearance of yellow chlorotic spots (leaf with $1 \%$ to $25 \%$ of attack symptoms); III = some yellow chlorotic spots and/or starting of black sooty mold (leaf with $26 \%$ to $50 \%$ of attack symptoms); IV = several yellow chlorotic spots and/or severe blackening of leaves (leaf with 51\% to 75\% of attack symptoms); and $\mathrm{V}=$ yellowing or complete drying leaves (leaf with $76 \%$ to $100 \%$ of attack symptoms) - were assessed visually, and all insects (e.g., Formicidae - eusocial insects) and spiders were counted, between 7:00 A.M. and 11:00 A.M., by visual observation, every two weeks on the adaxial and abaxial surfaces of the first 12 leaves expanded, per sapling. These leaves were 
assessed, randomly, on branches (one leaf per position) in the basal, middle, and apical parts of the canopy - vertical axis - (0 to $33 \%, 34$ to $66 \%$, and 67 to $100 \%$ of total sapling height, respectively) and in the north, south, east, and west directions - horizontal axis. A total of 12 leaves/sapling/ evaluation were observed on 48 A. auriculiformis saplings starting six months after transplantation for 24 months (27,648 total leaves), covering the entire sapling (vertical and horizontal axis), capturing the highest possible number of arthropods (insects and spiders), especially the rarest ones. The evaluator approached, carefully, firstly assessing the adaxial leaf surface and, if it was not possible to visualize the abaxial one, with a delicate and slow movement, the leaf was lifted and visualized. The position of leaves of A. auriculiformis saplings is generally tilted upwards, facilitating the visual assessment of arthropods on their leaf surfaces. Insects with greater mobility (e.g., Orthoptera), that flew, on approach, were counted as they were recognized (e.g., Order). The arthropods (insects and spiders) were not removed from the saplings during the evaluation.

A few arthropod specimens (up to 3 individuals) per species were collected with an aspirator (two hours per week), at the beginning of the study (between transplantation and first evaluation, six months after), stored in flasks with 70\% alcohol, separated into morph species, and sent to specialists for identification (see acknowledgments). Any visible arthropod, not yet computed in previous evaluations, was collected, coded and sent to a taxonomist of its group.

\subsection{Statistical analysis}

Each replication is the total of individuals collected on 12 leaves (three heights and four sides of the sapling) for 24 months. The type of distribution (aggregated, random, or regular) of lost source (L.S.) or solution source (S.S.) was defined by the Chi-square test using the BioDiversity Professional program, version 2 (Krebs, 1989) (Tables 1 and 2). The data were subjected to simple regression analysis and their parameters were all significant $(P<0.05)$ using the statistical program System for Analysis Statistics and Genetics (Saeg, 2007), version 9.1 (Table 3). Simple equations were selected by observing the criteria: i) distribution of data in the figures (linear or quadratic response), ii) the parameters used in these regressions were the most significant ones $(P<0.05)$, iii) $P<0.05$ and $F$ of the Analysis of Variance of these regressions, and iv) the coefficient of determination of these equations $\left(R^{2}\right)$. Only L.S. and S.S. with $P<0.05$ were shown in tables $1-3$. It is necessary knowledge of the system to select the possible loss sources and solution sources.

Percentage of Importance Indice-Production Unknown (\% I.I.-PU) was named because three other indexes, two with lower and one with higher knowledge of the system (Demolin-Leite, 2021), were created, but are not presented on this paper.

The developed formula is Equation 1:

$\%$ I.I. $-P U=\left[\left(k s_{1} x c_{1} x d s_{1}\right) / \Sigma\left(k s_{1} x c_{1} x d s_{1}\right)+\left(k s_{2} x c_{2} x d s_{2}\right)+\left(k s_{n} x c_{n} x d s_{n}\right)\right] x 100$,

where, i) key source ( $k s)$ is Equation 2:

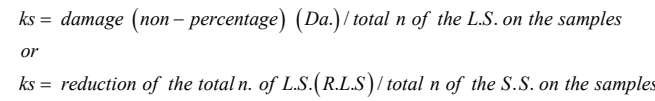

Where,

Da. or R.L.S. $=\mathrm{R}^{2} \mathrm{x}(1-P)$, when it is of the first degree, or $\left(\left(R^{2} \times(1-P)\right) \times\left(\beta_{2} / \beta_{1}\right)\right.$, when it is of the second degree, where $\mathbf{R}^{\mathbf{2}}=$ determination coefficient and $\boldsymbol{P}=$ significance of ANOVA, $\beta_{1}=$ regression coefficient, and $\beta_{2}=$ regression coefficient (variable ${ }^{2}$ ), of the simple regression equation of the loss source (L.S.) or solution source (S.S.).

When it is not possible to separate the $\mathrm{Da}$. between two or more L.S., divide the Da. among the L.S. in proportion to their respective "total $n$ ". $D a .=0$ when $\mathrm{Da}$. non-significant for damage or non-detected by L.S. on the system.

When a S.S. operates in more than one L.S., that caused damage, its $k$ s are summed. R.L.S. $=0$ when Da. by L.S. or R.L.S. non-significant for damage by L.S. or reduced L.S. by S.S. on the system.

ii) $c($ constancy $)=\Sigma$ of occurrence of L.S. or S.S. on samples, where,

absence $=0$ or presence $=1$.

iii) $d s$ (distribution source $)=1-\boldsymbol{P}$ of the chi-square test of L.S. or S.S. on the samples.

The Percentage of R.L.S. per S.S. is Equation 3:

Percentage of R.L.S. per S.S. $(\%$ R.L.S.S.S. $)=($ R.L.S.S.S. $/$ total $n$ of the L.S. - abundance or damage $) \times 100$

where,

R.L.S.S.S. $=$ R.L.S. $\mathrm{x}$ total $n$ of the S.S.,

In this case, the R.L.S. are not summed.

\section{Results}

The loss sources, per individual, Aethalion reticulatum L., 1767 (Hemiptera: Aethalionidae) (38.86\%), Aleyrodidae (Hemiptera) (37.69\%), Stereoma anchoralis Lacordaire, 1848 (Coleoptera: Chrysomelidae) (13.90\%), and Tettigoniidae (5.54\%), among 35 herbivorous insects ( $\approx 0.10 \%$ ), showed the highest \% I.I.-PU on leaves of $A$. auriculiformis saplings (Table 4).

The solution sources, per individual, Uspachus sp. (Araneae: Salticidae) (96.72\%), Salticidae (Araneae) (2.83\%), and Pseudomyrmex termitarius (Smith, 1877) (Hymenoptera: Formicidae) (0.45\%), among 24 natural enemies $(=0.00 \%)$, revealed the highest \% I.I.-PU on leaves of A. auriculiformis saplings. The number of Diabrotica speciosa Germar, 1824 (Coleoptera: Chrysomelidae) was reduced per number of Salticidae (13.85\%); that of A. reticulatum that of Uspachus sp. (1.01\%); and that of Cephalocoema sp. (Orthoptera: Proscopiidae) that of P. termitarius (23.54\%), totaling $38.40 \%$ of reduction of these herbivorous insects (numbers) on A. auriculiformis saplings. However, the number of Aleyrodidae was increased per number of Cephalotes sp. (Hymenoptera: Formicidae) (2.10\%) and that of $A$. reticulatum that of Brachymyrmex sp. (Hymenoptera: Formicidae) (93.01\%), totaling $95.11 \%$ of increase of these sap-sucking insects on A. auriculiformis saplings. The final balance was negative, with an increase of herbivorous 
Table 1. Aggregated (Agg.), regular (Reg.), or random (Ran.) distribution (Dist.) of the loss sources on 48 Acacia auriculiformis (Fabaceae) saplings.

\begin{tabular}{|c|c|c|c|c|c|}
\hline \multirow{2}{*}{$\begin{array}{c}\text { Loss } \\
\text { Sources }\end{array}$} & \multicolumn{5}{|c|}{ Qui-square test } \\
\hline & Var.* & Mean & C.sq..$^{\mathrm{f}}$ & $\boldsymbol{P}$ & Dist. \\
\hline Coleoptera: Cerambycidae & 0.02 & 0.02 & 44.00 & 0.47 & Ran. \\
\hline Chrysomelidae, Alagoasa sp. & 0.04 & 0.04 & 43.00 & 0.52 & Ran. \\
\hline Cerotoma sp. & 0.24 & 0.18 & 59.50 & 0.06 & Ran. \\
\hline Charidotis sp. & 0.02 & 0.02 & 44.00 & 0.47 & Ran. \\
\hline Diabrotica speciosa Germar, 1824 & 0.21 & 0.20 & 46.00 & 0.39 & Ran. \\
\hline Disonycha brasiliensis Costa Lima, 1954 & 0.04 & 0.04 & 43.00 & 0.51 & Ran. \\
\hline Eumolpus sp. & 0.04 & 0.04 & 43.00 & 0.52 & Ran. \\
\hline Parasyphraea sp. & 1.03 & 0.44 & 101.50 & 0.00 & Agg. \\
\hline Stereoma anchoralis Lacordaire, 1848 & 2.04 & 1.09 & 82.33 & 0.00 & Agg. \\
\hline Walterianela sp. & 0.04 & 0.04 & 43.00 & 0.52 & Ran. \\
\hline Curculionidae & 0.04 & 0.04 & 43.00 & 0.52 & Ran. \\
\hline Cratosomus sp. & 0.04 & 0.04 & 43.00 & 0.52 & Ran. \\
\hline Lordops sp. & 0.06 & 0.07 & 42.00 & 0.56 & Ran. \\
\hline Naupactus sp. & 0.06 & 0.07 & 42.00 & 0.56 & Ran. \\
\hline Tenebrionidae, Alleculinae & 0.02 & 0.02 & 44.00 & 0.47 & Ran. \\
\hline Hemiptera: Aethalionidae, Aethalion reticulatum L., 1767 & 71.05 & 2.76 & 1134.55 & 0.00 & Agg. \\
\hline Aleyrodidae & 8.65 & 0.82 & 462.87 & 0.00 & Agg. \\
\hline Cicadellidae, Achillidae & 0.09 & 0.04 & 88.00 & 0.00 & Agg. \\
\hline Acrogonia sp. & 0.02 & 0.02 & 44.00 & 0.47 & Ran. \\
\hline Balclutha hebe Kirkaldy, 1906 & 0.93 & 0.42 & 97.05 & 0.00 & Agg. \\
\hline Erythrogonia sexguttata Fabr., 1803 & 0.13 & 0.09 & 63.50 & 0.03 & Ran. \\
\hline Cicadidae, Quesada gigas Oliver, 1854 & 0.04 & 0.04 & 43.00 & 0.51 & Ran. \\
\hline Coreidae, Leptoglossus sp. & 0.02 & 0.02 & 44.00 & 0.47 & Ran. \\
\hline Fulgoridae & 0.02 & 0.02 & 44.00 & 0.47 & Ran. \\
\hline Membracidae & 0.10 & 0.11 & 40.00 & 0.64 & Ran. \\
\hline Membracis sp. & 2.60 & 0.82 & 139.35 & 0.00 & Agg. \\
\hline Nogodinidae & 0.02 & 0.02 & 44.00 & 0.47 & Ran. \\
\hline Pentatomidae & 0.62 & 0.53 & 51.00 & 0.22 & Ran. \\
\hline Scutelleridae, Pachycoris torridus Scopoli, 1772 & 0.70 & 0.38 & 80.94 & 0.00 & Agg. \\
\hline Hymenoptera: Apidae, Trigona spinipes Fabr., 1793 & 0.04 & 0.04 & 43.00 & 0.51 & Ran. \\
\hline Lepidoptera & 0.13 & 0.16 & 38.00 & 0.73 & Ran. \\
\hline Orthoptera: Proscopiidae, Cephalocoema sp. & 0.16 & 0.13 & 54.00 & 0.14 & Ran. \\
\hline Romaleidae, Tropidacris collaris Stoll., 1813 & 0.97 & 0.93 & 45.86 & 0.39 & Ran. \\
\hline Tettigoniidae & 0.61 & 0.73 & 36.55 & 0.78 & Ran. \\
\hline Phasmatodea: Phasmatidae, Phibalosoma phyllinum Gray, 1835 & 0.02 & 0.02 & 44.00 & 0.47 & Ran. \\
\hline
\end{tabular}

*= variance and $\mathrm{f}=$ chi-square.

insects of $56.71 \%$ in these saplings. The A. reticulatum damage, per individual, was reduced per number of Uspachus sp. (6.17\%); but the Aleyrodidae damage, per individual, was increased per number of Cephalotes sp. (30.00\%), totaling $23.81 \%$ of increase by insect damages on A. auriculiformis saplings (Tables 5 and 6).

\section{Discussion}

The Percentage of Importance Indice-Production Unknown (\% I.I.-PU) was effective in identifying loss (e.g., Tettigoniidae) and solution (e.g., Salticidae) sources and of the S.S. important on damage reduction by L.S. (e.g., 
Table 2. Aggregated (Agg.), regular (Reg.), or random (Ran.) distribution (Dist.) of the solution sources on 48 Acacia auriculiformis (Fabaceae) saplings.

\begin{tabular}{|c|c|c|c|c|c|}
\hline \multirow{2}{*}{$\begin{array}{l}\text { Solution } \\
\text { Sources }\end{array}$} & \multicolumn{5}{|c|}{ Qui-square test } \\
\hline & Var.* & Mean & C.sq. ${ }^{\mathrm{E}}$ & $\boldsymbol{P}$ & Dist. \\
\hline Araneae: Araneidae & 0.48 & 0.48 & 44.91 & 0.52 & Ran. \\
\hline Anyphaenidae, Teudis sp. & 0.16 & 0.13 & 55.33 & 0.14 & Ran. \\
\hline Oxyopidae & 0.24 & 0.17 & 61.00 & 0.06 & Ran. \\
\hline Oxyopes salticus Hentz, 1845 & 0.04 & 0.04 & 44.00 & 0.51 & Ran. \\
\hline Salticidae & 0.29 & 0.26 & 49.33 & 0.30 & Ran. \\
\hline Aphirape uncifera Tullgren, 1905 & 0.06 & 0.07 & 43.00 & 0.56 & Ran. \\
\hline Uspachus sp. & 0.14 & 0.11 & 59.40 & 0.07 & Ran. \\
\hline Sparassidae, Quemedice sp. & 0.06 & 0.07 & 43.00 & 0.56 & Ran. \\
\hline Tetragnathidae, Leucauge sp. & 0.02 & 0.02 & 45.00 & 0.47 & Ran. \\
\hline Thomisidae, Tmarus sp. & 0.08 & 0.09 & 42.00 & 0.60 & Ran. \\
\hline Coleoptera: Cantharidae, Cantharis sp. & 0.02 & 0.02 & 45.00 & 0.47 & Ran. \\
\hline Coccinellidae, Cycloneda sanguinea L., 1763 & 0.08 & 0.09 & 42.00 & 0.60 & Ran. \\
\hline Diptera: Dolichopodidae & 1.17 & 0.93 & 56.49 & 0.12 & Ran. \\
\hline Syrphidae, Syrphus sp. & 0.18 & 0.15 & 52.14 & 0.22 & Ran. \\
\hline Hymenoptera: Formicidae, Brachymyrmex sp. & 117.31 & 5.39 & 979.16 & 0.00 & Agg. \\
\hline Camponotus sp. & 13.00 & 4.07 & 143.86 & 0.00 & Agg. \\
\hline Cephalotes sp. & 0.87 & 0.20 & 200.56 & 0.00 & Agg. \\
\hline Ectatoma sp. & 1.88 & 0.83 & 102.42 & 0.00 & Agg. \\
\hline Pheidole sp. & 21.08 & 3.63 & 261.32 & 0.00 & Agg. \\
\hline Pseudomyrmex termitarius (Smith, 1877) & 3.88 & 1.35 & 129.42 & 0.00 & Agg. \\
\hline Vespidae, Polybia sp. & 0.61 & 0.52 & 52.67 & 0.20 & Ran. \\
\hline Hemiptera: Pentatomidae, Podisus sp. & 0.11 & 0.07 & 73.67 & 0.00 & Agg. \\
\hline Mantodea: Mantidae, Mantis religiosa L., 1758 & 0.04 & 0.04 & 44.00 & 0.51 & Ran. \\
\hline Neuroptera: Chrysopidae, Chrysoperla sp. & 0.04 & 0.04 & 44.00 & 0.51 & Ran. \\
\hline
\end{tabular}

${ }^{*}=$ variance and $^{\mathrm{f}}=$ chi-square.

Table 3. Simple regression equations of damage per loss source (L.S.) and reduction or increase of L.S. (abundance or damage) per solution source (S.S.) on 48 Acacia auriculiformis (Fabaceae) saplings.

\begin{tabular}{|c|c|c|c|}
\hline \multirow{2}{*}{ Simple regression analysis } & \multirow{2}{*}{$\mathbf{R}^{2}$} & \multicolumn{2}{|c|}{ ANOVA } \\
\hline & & $\boldsymbol{F}$ & $\boldsymbol{P}$ \\
\hline Defoliation $=4.02-0.33 \times$ Stereoma anchoralis $+0.13 \times$ Stereoma anchoralis $^{2}$ & 0.20 & 5.46 & 0.0075 \\
\hline Defoliation=4.22-1.03xTettigoniidae $+0.51 \times$ Tettigoniidae $^{2}$ & 0.18 & 4.98 & 0.0111 \\
\hline Damage $=-0.03+0.08 x$ Aleyrodidae & 0.98 & 1881.6 & 0.0000 \\
\hline Damage $=-0.0005+0.19 x$ Aethalion reticulatum & 0.99 & 3217.0 & 0.0000 \\
\hline Aleyrodidae damage $=0.11+0.42 \times$ Cephalotes $\mathrm{sp}$. & 0.30 & 19.41 & 0.0001 \\
\hline A. reticulatum damage $=0.09+1.86 \mathrm{x}$ Uspachus sp. $-0.96 \mathrm{x}$ Uspachus sp. ${ }^{2}$ & 0.17 & 4.53 & 0.0161 \\
\hline Aethalion reticulatum $=0.50+9.25 \mathrm{x}$ Uspachus sp. $-4.75 \mathrm{x}$ Uspachus sp. ${ }^{2}$ & 0.15 & 3.98 & 0.0256 \\
\hline Aethalion reticulatum $=0.25+0.10 \times$ Brachymyrmex sp. & 0.14 & 7.50 & 0.0088 \\
\hline Aleyrodidae $=1.69+4.78 \times$ Cephalotes sp. & 0.29 & 18.32 & 0.0001 \\
\hline Diabrotica specios $a=0.11+1.09 \times$ Salticidae- $0.57 \times x_{\text {Salticidae }}^{2}$ & 0.20 & 5.61 & 0.0067 \\
\hline Cephalocoema sp. $=-0.01+0.22 \times P$. termitarius $-0.03 \times P$. termitarius ${ }^{2}$ & 0.17 & 4.45 & 0.0173 \\
\hline
\end{tabular}


Table 4. Total number $(n)$, damage ( $D a$.), key-source $(k s)$, constancy $(c)$, distribution source $(d s)$, number of importance indice (n. I.I.), sum of n. I.I.-PU ( $\Sigma$ n. I.I. ), and percentage of I.I. by loss source (L.S.) on 48 Acacia auriculiformis (Fabaceae) saplings.

\begin{tabular}{|c|c|c|c|c|c|c|c|c|}
\hline \multicolumn{9}{|c|}{ Loss source } \\
\hline L.S. & $n$ & Da. & ks & $c$ & ds & n. I.I. & $\Sigma$ n. I.I. & \% I.I. \\
\hline Aethalion reticulatum & 37 & 0.9800 & 0.0268 & 4 & 1.00 & 0.1070 & 0.28 & 38.86 \\
\hline Aleyrodidae & 124 & 0.9800 & 0.008 & 13 & 1.00 & 0.1038 & 0.28 & 37.69 \\
\hline Stereoma anchoralis & 49 & 0.0782 & 0.0016 & 24 & 1.00 & 0.0383 & 0.28 & 13.90 \\
\hline Tettigoniidae & 33 & 0.0881 & 0.0027 & 26 & 0.22 & 0.0153 & 0.28 & 5.54 \\
\hline Tropidacris collaris & 42 & 0.0087 & 0.0002 & 26 & 0.61 & 0.0032 & 0.28 & 1.18 \\
\hline Parasyphraea sp. & 20 & 0.0041 & 0.0002 & 11 & 1.00 & 0.0023 & 0.28 & 0.82 \\
\hline Cerotoma sp. & 8 & 0.0017 & 0.0002 & 6 & 0.94 & 0.0012 & 0.28 & 0.42 \\
\hline Diabrotica speciosa & 9 & 0.0019 & 0.0002 & 8 & 0.61 & 0.0010 & 0.28 & 0.37 \\
\hline Cephalocoema sp. & 6 & 0.0012 & 0.0002 & 5 & 0.86 & 0.0009 & 0.28 & 0.32 \\
\hline Lepidoptera & 7 & 0.0014 & 0.0002 & 7 & 0.27 & 0.0004 & 0.28 & 0.14 \\
\hline Lordops sp. & 3 & 0.0006 & 0.0002 & 3 & 0.44 & 0.0003 & 0.28 & 0.10 \\
\hline Naupactus sp. & 3 & 0.0006 & 0.0002 & 3 & 0.44 & 0.0003 & 0.28 & 0.10 \\
\hline Alagoasa sp. & 2 & 0.0004 & 0.0002 & 2 & 0.49 & 0.0002 & 0.28 & 0.07 \\
\hline Disonycha brasiliensis & 2 & 0.0004 & 0.0002 & 2 & 0.49 & 0.0002 & 0.28 & 0.07 \\
\hline Eumolpus sp. & 2 & 0.0004 & 0.0002 & 2 & 0.49 & 0.0002 & 0.28 & 0.07 \\
\hline Walterianela sp. & 2 & 0.0004 & 0.0002 & 2 & 0.49 & 0.0002 & 0.28 & 0.07 \\
\hline Cratosomus sp. & 2 & 0.0004 & 0.0002 & 2 & 0.49 & 0.0002 & 0.28 & 0.07 \\
\hline Curculionidae & 2 & 0.0004 & 0.0002 & 2 & 0.49 & 0.0002 & 0.28 & 0.07 \\
\hline Charidotis sp. & 1 & 0.0002 & 0.0002 & 1 & 0.53 & 0.0001 & 0.28 & 0.04 \\
\hline Alleculinae & 1 & 0.0002 & 0.0002 & 1 & 0.53 & 0.0001 & 0.28 & 0.04 \\
\hline Phibalossoma phyllinum & 1 & 0.0002 & 0.0002 & 1 & 0.53 & 0.0001 & 0.28 & 0.04 \\
\hline Cerambycidae & 1 & 0.0000 & 0.0000 & 1 & 0.53 & 0.0000 & 0.28 & 0.00 \\
\hline Achillidae & 2 & 0.0000 & 0.0000 & 1 & 1.00 & 0.0000 & 0.28 & 0.00 \\
\hline Acrogonia sp. & 1 & 0.0000 & 0.0000 & 1 & 0.53 & 0.0000 & 0.28 & 0.00 \\
\hline Balclutha hebe & 19 & 0.0000 & 0.0000 & 10 & 1.00 & 0.0000 & 0.28 & 0.00 \\
\hline Erythrogonia sexguttata & 4 & 0.0000 & 0.0000 & 3 & 0.97 & 0.0000 & 0.28 & 0.00 \\
\hline Quesada gigas & 2 & 0.0000 & 0.0000 & 2 & 0.49 & 0.0000 & 0.28 & 0.00 \\
\hline Leptoglossus sp. & 1 & 0.0000 & 0.0000 & 1 & 0.53 & 0.0000 & 0.28 & 0.00 \\
\hline Fulgoridae & 1 & 0.0000 & 0.0000 & 1 & 0.53 & 0.0000 & 0.28 & 0.00 \\
\hline Membracidae & 5 & 0.0000 & 0.0000 & 5 & 0.36 & 0.0000 & 0.28 & 0.00 \\
\hline Membracis sp. & 37 & 0.0000 & 0.0000 & 19 & 1.00 & 0.0000 & 0.28 & 0.00 \\
\hline Nogodinidae & 1 & 0.0000 & 0.0000 & 1 & 0.53 & 0.0000 & 0.28 & 0.00 \\
\hline Pentatomidae & 24 & 0.0000 & 0.0000 & 19 & 0.78 & 0.0000 & 0.28 & 0.00 \\
\hline Pachycoris torridus & 17 & 0.0000 & 0.0000 & 9 & 1.00 & 0.0000 & 0.28 & 0.00 \\
\hline Trigona spinipes & 2 & 0.0000 & 0.0000 & 2 & 0.49 & 0.0000 & 0.28 & 0.00 \\
\hline
\end{tabular}

I.I. $-P U=k s \times c \times d s . k s=D a$. $/$ total $n$ of the L.S.. Da. $=\mathrm{R}^{2} \mathrm{x}(1-P)$ when it is of the first degree, or $\left(\left(\mathrm{R}^{2} \mathrm{x}(1-P)\right) \mathrm{x}\left(\beta_{2} / \beta_{1}\right)\right.$ when it is of the second degree, where $\mathbf{R}^{2}=$ determination coefficient and $\boldsymbol{P}=$ significance of ANOVA, $\beta_{1}=$ regression coefficient, and $\beta_{2}=$ regression coefficient $\left(\right.$ variable ${ }^{2}$ ), of the simple regression equation, or non-percentage of damage per L.S.. $c=\Sigma$ of occurrence of L.S. on each sample, $0=$ absence or $1=$ presence. $d s=1-\boldsymbol{P}$ of chi-square test of the L.S.. Da. $=0$ when Da. non-significant for damage or non-detected by L.S.

Uspachus sp. versus A. reticulatum) on a system with production unknown (e.g., A. auriculiformis saplings).

The loss sources A. reticulatum, Aleyrodidae, S. anchoralis, and Tettigoniidae presented the highest \% I.I.-PU on leaves of A. auriculiformis saplings. These herbivorous insects are related as a pest in some crops. The $A$. reticulatum is a pest that reduces the development of fruits and sprouts, leading to hypertrophy and cracks in the apex of 
Table 5. Total number ( $n$ ), reduction of L.S. (R.L.S.), key-source $(k s)$, constancy $(c)$, distribution source ( $d s)$, number of importance indice (n. I.I.), sum of n. I.I.-PU ( $\Sigma$ n. I.I.), and percentage of I.I. by solution source (S.S.) on 48 Acacia auriculiformis (Fabaceae) saplings.

\begin{tabular}{|c|c|c|c|c|c|c|c|c|}
\hline \multicolumn{9}{|c|}{ Solution source } \\
\hline S.S. & $\boldsymbol{n}$ & R.L.S. & ks & c & ds & n. I.I. & $\Sigma$ n. I.I. & \% I.I. \\
\hline Uspachus sp. & 5 & 2.7800 & 0.5560 & 4 & 0.93 & 2.06 & 2.13 & 96.72 \\
\hline Salticidae & 12 & 0.1039 & 0.0087 & 10 & 0.70 & 0.06 & 2.13 & 2.83 \\
\hline Pseudomyrmex termitarius & 62 & 0.0228 & 0.0004 & 26 & 1.00 & 0.01 & 2.13 & 0.45 \\
\hline Araneidae & 22 & 0.0000 & 0.0000 & 17 & 0.48 & 0.00 & 2.13 & 0.00 \\
\hline Teudis sp. & 6 & 0.0000 & 0.0000 & 5 & 0.86 & 0.00 & 2.13 & 0.00 \\
\hline Oxyopidae & 8 & 0.0000 & 0.0000 & 6 & 0.94 & 0.00 & 2.13 & 0.00 \\
\hline Oxyopes salticus & 2 & 0.0000 & 0.0000 & 2 & 0.49 & 0.00 & 2.13 & 0.00 \\
\hline Aphirape uncifera & 3 & 0.0000 & 0.0000 & 3 & 0.44 & 0.00 & 2.13 & 0.00 \\
\hline Quemedice sp. & 3 & 0.0000 & 0.0000 & 3 & 0.44 & 0.00 & 2.13 & 0.00 \\
\hline Leucauge sp. & 1 & 0.0000 & 0.0000 & 1 & 0.53 & 0.00 & 2.13 & 0.00 \\
\hline Tmarus sp. & 4 & 0.0000 & 0.0000 & 4 & 0.40 & 0.00 & 2.13 & 0.00 \\
\hline Cantharis sp. & 1 & 0.0000 & 0.0000 & 1 & 0.53 & 0.00 & 2.13 & 0.00 \\
\hline Cycloneda sanguinea & 4 & 0.0000 & 0.0000 & 4 & 0.40 & 0.00 & 2.13 & 0.00 \\
\hline Dolichopodidae & 43 & 0.0000 & 0.0000 & 27 & 0.88 & 0.00 & 2.13 & 0.00 \\
\hline Syrphus $s p$. & 7 & 0.0000 & 0.0000 & 6 & 0.78 & 0.00 & 2.13 & 0.00 \\
\hline Brachymyrmex sp. & 248 & 0.0000 & 0.0000 & 28 & 1.00 & 0.00 & 2.13 & 0.00 \\
\hline Camponotus sp. & 187 & 0.0000 & 0.0000 & 41 & 1.00 & 0.00 & 2.13 & 0.00 \\
\hline Cephalotes sp. & 9 & 0.0000 & 0.0000 & 3 & 1.00 & 0.00 & 2.13 & 0.00 \\
\hline Ectatoma sp. & 38 & 0.0000 & 0.0000 & 18 & 1.00 & 0.00 & 2.13 & 0.00 \\
\hline Pheidole sp. & 167 & 0.0000 & 0.0000 & 41 & 1.00 & 0.00 & 2.13 & 0.00 \\
\hline Polybia sp. & 24 & 0.0000 & 0.0000 & 18 & 0.80 & 0.00 & 2.13 & 0.00 \\
\hline Podisus sp. & 3 & 0.0000 & 0.0000 & 2 & 1.00 & 0.00 & 2.13 & 0.00 \\
\hline Mantis religiosa & 2 & 0.0000 & 0.0000 & 2 & 0.49 & 0.00 & 2.13 & 0.00 \\
\hline Chrysoperla sp. & 2 & 0.0000 & 0.0000 & 2 & 0.49 & 0.00 & 2.13 & 0.00 \\
\hline
\end{tabular}

I.I. $-P U=k s \times \mathrm{x}$ x ds. ks = R.L.S./total $n$. of the S.S.. R.L.S. $=\mathrm{R}^{2} \mathrm{x}(1-P)$ when it is of the first degree, or $\left(\left(\mathrm{R}^{2} \mathrm{x}(1-P)\right) \mathrm{x}\left(\beta_{2} / \beta_{1}\right)\right.$ when it is of the second degree, where $\mathbf{R}^{2}=$ determination coefficient and $\boldsymbol{P}=$ significance of ANOVA, $\beta_{1}=$ regression coefficient, and $\beta_{2}=$ regression coefficient (variable ${ }^{2}$, of the simple regression equation. $c=\Sigma$ of occurrence of $S . S$. on each sample, $0=$ absence or $1=$ presence. $d s=1-\boldsymbol{P}$ of chi-square test of the S.S. When a S.S. operates in more than one L.S., that caused damage, its ks are summed. E.S.. = 0 when Da. by L.S. or E.S. non-significant for damage by L.S. or reduced L.S. by S.S.

seedlings and possibly killing plants of "mulungu" Erythrina speciosa Andrews (Fabales: Fabaceae) (Araújo et al., 2010; Zanuncio et al., 2015). In addition, this sap-sucking insect damages A. mangium, ant tree Triplaris americana $\mathrm{L}$. (Caryophyllales: Polygonaceae), and bitterleaf Vernonia condensata Baker (Asterales: Asteraceae) (De Menezes et al., 2013; Pires et al., 2015; Silva et al., 2020; Gomes et al., 2023; Lima et al., 2024). Aleyrodidae family has Bemisia tabaci (Genn., 1889), as a pest of several plants, including sweet pepper Capsicum annuum L. (Solanales: Solanaceae), melon Cucumis melo L. (Cucurbitales: Cucurbitaceae), soybean Glycine max (L.) Merrill (Fabales: Fabaceae) and common bean Phaseolus vulgaris L. (Fabales: Fabaceae), and tomato Solanum lycopersicon Mill. (Solanales: Solanaceae) by sucking sap, injecting toxins, transmitting viruses, and favors the development of fumagine (Zhang et al., 2004; Mansaray and Sundufu, 2009; Kim et al., 2017; Felicio et al.,
2019). The species S. anchoralis damages A. mangium and leucaena Leucaena leucocephala (Lam.) de Wit (Fabales: Fabaceae) trees (Damascena et al., 2017; Silva et al., 2020), and Meroncidius intermedius (Brunner von Wattenwyl, 1895) (Orthoptera: Tettigoniidae) damaged grasses and banana Musa spp. fruits (Zingiberales: Musaceae) (Zanuncio-Junior et al., 2017).

The solution sources Uspachus sp., Salticidae, and P. termitarius showed the highest \% I.I.-PU on leaves of A. auriculiformis saplings. The numbers of D. speciosa, A. reticulatum, and Cephalocoema sp. were reduced per numbers of Salticidae, Uspachus sp. (also reduced damage by this pest, $\approx 6 \%$ ), and $P$. termitarius, respectively, on $A$. auriculiformis saplings. Spiders are important predators, as an example, on A. mangium and pequi tree Caryocar brasiliense Camb. (Malpighiales: Caryocaraceae) trees, in the Brazilian Cerrado (Leite et al., 2012a; Silva et al., 
Table 6. Percentage of reduction in abundance and/or damage (\%R.) of loss source (L.S.) per solution source (S.S.), sum ( $\Sigma$ ), and total of $\Sigma$ of R.L.S. (T. $\Sigma$ ) on 48 Acacia auriculiformis (Fabaceae) saplings.

\begin{tabular}{|c|c|c|c|c|}
\hline \multirow[b]{3}{*}{ S.S. } & \multicolumn{4}{|c|}{ \%R.L.S.S.S.- abundance } \\
\hline & \multicolumn{4}{|c|}{ L.S. } \\
\hline & D. speciosa & A. reticulatum & Aleyrodidae & Cephalocoema sp. \\
\hline Salticidae & 13.85 & --- & --- & --- \\
\hline Uspachus sp. & --- & 1.01 & --- & --- \\
\hline Brachymyrmex sp. & --- & -93.01 & --- & --- \\
\hline Cephalotes sp. & --- & --- & -2.10 & --- \\
\hline P. termitarius & --- & --- & --- & 23.54 \\
\hline$\Sigma$ & 13.85 & -92.00 & -2.10 & 23.54 \\
\hline \multirow[t]{3}{*}{ T. $\Sigma$} & -56.71 & --- & --- & --- \\
\hline & \multicolumn{4}{|c|}{ \%R.L.S.S.S.- damage } \\
\hline & \multicolumn{4}{|c|}{ L.S. } \\
\hline S.S. & D. speciosa & A. reticulatum & Aleyrodidae & Cephalocoema sp. \\
\hline Uspachus sp. & --- & 6.17 & --- & --- \\
\hline Cephalotes sp. & --- & --- & -30.00 & --- \\
\hline$\Sigma$ & --- & 6.17 & -30.00 & --- \\
\hline T. $\Sigma$ & -23.83 & --- & --- & --- \\
\hline
\end{tabular}

--- = L.S. was not reduced per S.S. \%R.L.S.S.S. $=($ R.L.S.S.S. $/$ total $n$ of the L.S. - abundance or damage $) \mathrm{x} 100$, where R.L.S.S.S. $=$ R.L.S. $\mathrm{x}$ total $\mathrm{n}$ of the S.S. R.L.S. $=\mathrm{R}^{2} \mathrm{x}(1-P)$ when it is of the first degree, or $\left(\left(\mathrm{R}^{2} \mathrm{x}(1-P)\right) \mathrm{x}\left(\beta_{2} / \beta_{1}\right)\right.$ when it is of the second degree, where $\mathbf{R}^{2}=$ determination coefficient and $\boldsymbol{P}=$ significance of ANOVA, $\beta_{1}=$ regression coefficient, and $\beta_{2}=$ regression coefficient (variable ${ }^{2}$ ), of the simple regression equation.

2020); on pastures and forests in Greece, and in $A$. auriculiformis saplings in a degraded area in Brazil, being directly correlated with Orthoptera (Zografou et al., 2017; Mota et al., 2023); in many agroecosystems in the USA (Landis et al., 2000) and Italy (Venturino et al., 2008); and in 12 agricultural landscapes in the low mountain ranges of Central Hesse (Germany)(Öberg et al., 2008). Moreover, ants can reduce defoliation and fruit-boring insect populations (e.g., Coleoptera and Lepidoptera) (Leite et al., 2012a; Gonthier et al., 2013; Fagundes et al., 2017, Dassou et al. 2019) besides, they are bioindicators of the recovery of degraded areas (Sanchez, 2015). However, the numbers of Aleyrodidae and $A$. reticulatum were increased per numbers of Cephalotes sp. (increasing Aleyrodidae damage $\approx 30 \%$ ) and Brachymyrmex sp., respectively, totaling, $\approx 95 \%$ of increase of these sap-sucking insects on A. auriculiformis saplings. These facts can be a problem in A. auriculiformis commercial crops. Sap-sucking insects, especially at high densities, can be associated with ants (mutual benefit), showing a direct correlation between these groups (Leite et al., 2012b, 2015, 2016; Novgorodova, 2015; Sanchez et al., 2020) because they collectively and aggressively defend their resources (e.g., sap-sucking insects) (Novgorodova, 2015). Dominant ants form mutualistic relationships with sap-sucking insects, with the negative impact of the latter on the biological control of sap-sucking hemipterans (Karami-Jamour et al., 2018; Tong et al., 2019). This relationship increases pest problems in agricultural systems (Sagata and Gibb, 2016).

\section{Conclusions}

The loss sources A. reticulatum, Aleyrodidae, S. anchoralis, and Tettigoniidae showed the highest \% I.I.-PU on leaves of A. auriculiformis saplings. These insects turn into problems on A. auriculiformis plantations since they are related to pests in some crops. The solution sources Uspachus sp., Salticidae, and P. termitarius showed the highest \% I.I.-$P U$ on leaves of $A$. auriculiformis saplings. These natural enemies can be important to $A$. auriculiformis because they can reduce herbivorous damages (e.g., A. reticulatum damage versus Uspachus sp.). However, ants Cephalotes sp. and Brachymyrmex sp. increased around $95 \%$ of Aleyrodidae and A. reticulatum populations and can be a problem in A. auriculiformis commercial crops. Here I showed and tested the \%III. $-P U$, a new index that can detect the loss or solution sources, when production is unknown, on a system, and it can be applied in various knowledge areas.

\section{Acknowledgments}

I wish to thank the taxonomists Dr. Antônio Domingos Brescovit (Butantan Institute, São Paulo State, Brazil - Arachnida), Dr. Ayr de Moura Bello (Oswaldo Cruz Foundation, Rio de Janeiro State, Brazil - Coleoptera), Dr. Carlos Matrangolo (University of Montes Claros, Minas Gerais State, Brazil - Formicidae), Dr. Ivan Cardoso Nascimento (EMBRAPA-ILHÉUS Cocoa Research Center, CEPLAC, Itabuna, Bahia State, Brazil - Formicidae), Dr. Luci 
Boa Nova Coelho (Federal University of Rio de Janeiro, Rio de Janeiro State, Brazil - Cicadellidae), and Dr. Paulo Sérgio Fiuza Ferreira (Federal University of Viçosa, Minas Gerais State, Brazil - Hemiptera) for the identification of specimens. The voucher numbers are 1595/02 and 1597/02 (CDZOO, Federal University of Paraná, Paraná State, Brazil).

\section{References}

AGUIAR JUNIOR, A., BARBOSA, R.I., BARBOSA, J.B.F. and MOURÃO JUNIOR, M., 2014. Invasion of Acacia mangium in Amazonian savannas following planting for forestry. Plant Ecology $\mathcal{E}$ Diversity, vol. 7, no. 1-2, pp. 359-369. http://dx.doi.org/10.108 0/17550874.2013.771714.

ALVARES, C.A., STAPE, J.L., SENTELHAS, P.C., DE MORAES GONÇALVES, J.L. and SPAROVEK, G., 2013. Köppen's climate classification map for Brazil. Meteorologische Zeitschrift (Berlin), vol. 22, no. 6, pp. 711-728. http://dx.doi.org/10.1127/0941-2948/2013/0507.

ARAÚJO, V.A., BÁO, S.N., MOREIRA, J., NEVES, C.A. and LINO-NETO, J., 2010. Ultrastructural characterization of the spermatozoa of Aethalion reticulatum Linnaeus 1767 (Hemiptera: Auchenorrhyncha: Aethalionidae). Micron (Oxford, England : 1993), vol. 41, no. 4, pp. 306-311. http://dx.doi.org/10.1016/j. micron.2009.12.001. PMid:20047835.

BALIEIRO, F.C., COSTA, C.A., OLIVEIRA, R.B., DONAGEMMA, G.K., ANDRADE, A.G. and CAPECHE, C.L., 2017. Carbon stocks in mined area reclaimed by leguminous trees and sludge. Revista Árvore, vol. 41, e410610. https://doi.org/10.1590/180690882017000600010.

DA SILVA, E.M., DA SILVA, R.S., RODRIGUES-SILVA, N., MILAGRES, C.C., BACCI, L. and PICANÇO, M.C., 2017. Assessment of the natural control of Neoleucinodes elegantalis in tomato cultivation using ecological life tables. Biocontrol Science and Technology, vol. 27, no. 4, pp. 1-14. http://dx.doi.org/10.1080/09583157.2017.1319911.

DAMASCENA, J.G., LEITE, G.L.D., SILVA, F.W.S., SOARES, M.A., GUAÑABENS, R.E.M., SAMPAIO, R.A. and ZANUNCIO, J.C., 2017. Spatial distribution of phytophagous insects, natural enemies, and pollinators on Leucaena leucocephala (Fabales: Fabaceae) trees in the Cerrado. Florida Entomologist, vol. 100, no. 3, pp. 558-565. https://doi.org/10.1653/024.100.0311.

DASSOU, A.G., VODOUHÉ, S.D., BOKONON-GANTA, A., GOERGEN, G., CHAILLEUX, A., DANSI, A., CARVAL, D. and TIXIER, P., 2019. Associated cultivated plants in tomato cropping systems structure arthropod communities and increase the Helicoverpa armigera regulation. Bulletin of Entomological Research, vol. 109, no. 6, pp. 733-740. http://dx.doi.org/10.1017/ S0007485319000117. PMid:30968787.

DAVIS, S.K. and WIGELSWORTH, M., 2018. Structural and predictive properties of the emotional quotient inventory youth versionshort form (EQ-i:YV[S]). Journal of Personality Assessment, vol. 100, no. 2, pp. 197-206. http://dx.doi.org/10.1080/00223891.2 017.1280502. PMid:28631978.

DE MENEZES, C.W.G., BERTOLUCCI, S.K.V., PINTO, J.E.B.P., CARVALHO, G.A. and SOARES, M.A., 2013. First record of Aethalion reticulatum (Hemiptera: Aethalionidae) in Vernonia condensata (Asteraceae), a medicinal plant from Brazil. Phytoparasitica, vol. 41, no. 5, pp. 611-613. http://dx.doi.org/10.1007/s12600-013-0322-0.

DEMOLIN-LEITE, G.L., 2021. Importance indice: loss estimates and solution effectiveness on production. Cuban Journal of Agricultural Science, vol.55, no. 2, pp. 1-7. Available from: http:// scielo.sld.cu/pdf/cjas/v55n2/2079-3480-cjas-55-02-e10.pdf.
DORAN, J.C. and TURNBULL, J.W., 1997. Australian trees and shrubs: species for land rehabilitation and farm planting in the tropics. Bruce, Australian: ACIAR, pp. 384.

FAGUNDES, R., DÁTTILO, W., RIBEIRO, S.P., RICO-GRAY, V., JORDANO, P. and DEL-CLARO, K., 2017. Differences among ant species in plant protection are related to production of extrafloral nectar and degree of leaf herbivory. Biological Journal of the Linnean Society. Linnean Society of London, vol. 122, no. 1, pp. 71-83. http://dx.doi.org/10.1093/biolinnean/blx059.

FELICIO, T.N.P., COSTA, T.L., SARMENTO, R.A., RAMOS, R.S., PEREIRA, P.S., DA SILVA, R.S. and PICANÇO, M.C., 2019. Surrounding vegetation, climatic elements, and predators affect the spatial dynamics of Bemisia tabaci (Hemiptera: Aleyrodidae) in commercial melon fields. Journal of Economic Entomology, vol. 112, no. 6, pp. 2774-2781. http://dx.doi.org/10.1093/jee/ toz181. PMid:31265728.

FIRMANSYAH, M.A., ERFIANI, E., JAYANEGARA, A., ACHMAD, A. and WIJAYANTO, N., 2020. In vitro biological control of Ceratobasidium ramicola by using tannin extracts from Acacia villosa, Myristica fragrans, Acacia mangium, and Calliandra calothyrsus leaves. Brazilian Journal of Biology $=$ Revista Brasileira de Biologia, vol. 80, no. 2, pp. 235-239. http://dx.doi. org/10.1590/1519-6984.184912. PMid:31482970.

GOLDENBERG, M.G. and GRANTCHAROV, T.P., 2019. A novel method of setting performance standards in surgery using patient outcomes. Annals of Surgery, vol. 269, no. 1, pp. 79-82. http:// dx.doi.org/10.1097/SLA.0000000000002562. PMid:29064892.

GOMES, G.N., LEITE, G.L.D., SOARES, M.A., GUANÃBENS, R.E.M., LEMES, P.G. and ZANUNCIO, J.C., 2023. Arthropod fauna on the abaxial and adaxial surfaces of Acacia mangium (Fabaceae) leaves. Brazilian Journal of Biology = Revista Brasileira de Biologia, vol. 83, pp. e245536. http://dx.doi.org/10.1590/1519-6984.245536. PMid:34669792.

GONTHIER, D.J., ENNIS, K.K., PHILPOTT, S.M., VANDERMEER, J. and PERFECTO, I., 2013. Ants defend coffee from berry borer colonization. BioControl, vol. 58, no. 6, pp. 815-820. http:// dx.doi.org/10.1007/s10526-013-9541-z.

KARAMI-JAMOUR, T., MIRMOAYEDI, A., ZAMANI, A. and KHAJEHZADEH, Y., 2018. The impact of ant attendance on protecting Aphis gossypii against two aphidophagous predators and it's role on the intraguild predation between them. Journal of Insect Behavior, vol. 31, no. 2, pp. 222-239. http://dx.doi. org/10.1007/s10905-018-9670-4.

KIM, S., JUNG, M., SONG, Y.J., KANG, C., KIM, B.Y., CHOI, I.J., KIM, H.G. and LEE, D.H., 2017. Evaluating the potential of the extract of Perilla sp. as a natural insecticide for Bemisia tabaci (Hemiptera: Aleyrodidae) on sweet peppers. Entomological Research, vol. 47, no. 3, pp. 208-216. http://dx.doi.org/10.1111/1748-5967.12211.

KREBS, C.J., 1989 [accessed 2 May 2018]. Bray-Curtis cluster analysis [online]. Available from: http://biodiversity-pro.software. informer.com

LANDIS, D., WRATTEN, S.D. and GURR, G.M., 2000. Habitat management to conserve natural enemies of arthropod pests in agriculture. Annual Review of Entomology, vol. 45, no. 1, pp. 175-201. http://dx.doi.org/10.1146/annurev.ento.45.1.175. PMid:10761575.

LEITE, G.L.D., VELOSO, R.V.S., ZANUNCIO, J.C., ALMEIDA, C.I.M., FERREIRA, P.S.F., FERNANDES, G.W. and SOARES, M.A., 2012a. Habitat complexity and Caryocar brasiliense herbivores (Insecta; Arachnida; Araneae). The Florida Entomologist, vol. 95, no. 4, pp. 819-830. http://dx.doi.org/10.1653/024.095.0402.

LEITE, G.L.D., VELOSO, R.V.S., ZANUNCIO, J.C., FERNANDES, G.W., ALMEIDA, C.I.M., FERREIRA, P.S.F., ALONSO, J. and SERRÃO, 
J.E., 2012b. Seasonal abundance of hemipterans on Caryocar brasiliense (Malpighiales: Caryocaraceae) trees in the Cerrado. The Florida Entomologist, vol. 95, no. 4, pp. 862-872. http:// dx.doi.org/10.1653/024.095.0407.

LEITE, G.L.D., VELOSO, R.V.S., ZANUNCIO, J.C., FERNANDES, G.W., ALMEIDA, C.I.M., FERREIRA, P.S.F., ALONSO, J. and SERRÃO, J.E., 2015. Cardinal distribution of sucking insects in Caryocar brasiliense (Caryocaraceae) in Cerrado (Brazil). Revista Colombiana de Entomologia, vol. 41, no. 1, pp. 105-111.

LEITE, G.L.D., VELOSO, R.V.S., ZANUNCIO, J.C., ALONSO, J., FERREIRA, P.S.F., ALMEIDA, C.I.M., FERNANDES, G.W. and SERRÃO, J.E., 2016. Diversity of Hemiptera (Arthropoda: Insecta) and their natural enemies on Caryocar brasiliense (Malpighiales: Caryocaraceae) trees in the Brazilian Cerrado. The Florida Entomologist, vol. 99, no. 2, pp. 239-247. http://dx.doi.org/10.1653/024.099.0213.

LIMA, J.S., LEITE, G.L.D., GUANABENS, P.F.S., SOARES, M.A., SILVA, J.L., MOTA, M.V.S., LEMES, P.G. and ZANUNCIO, J.C., 2024. Insects and spiders on Acacia mangium (Fabaceae) saplings as bioindicators for the recovery of tropical degraded areas. Brazilian Journal of Biology = Revista Brasileira de Biologia, vol 84, pp. e252088. PMid:34755814.

LIN, S.J., LU, I.J. and LEWIS, C., 2007. Grey relation performance correlations among economics, energy use and carbon dioxide emission in Taiwan. Energy Policy, vol. 35, no. 3, pp. 1948-1955. http://dx.doi.org/10.1016/j.enpol.2006.06.012.

LIU, Y., CHEN, Y. and TZENG, G.H., 2017. Identification of key factors in consumers' adoption behavior of inteligente medical terminals based on a hybrid modified MADM model for product improvement. International Journal of Medical Informatics, vol. 105, pp. 68-82. http://dx.doi.org/10.1016/j.ijmedinf.2017.05.017. PMid:28750913.

LONDE, V., SOUSA, H.C. and MESSIAS, M.C.T.B., 2020. Monitoring of forest components reveals that exotic tree species are not always invasive in areas under ecological restoration. Environmental Monitoring and Assessment, vol. 192, no. 10, pp. 618-628. http:// dx.doi.org/10.1007/s10661-020-08583-w. PMid:32880735.

MANSARAY, A. and SUNDUFU, A.J., 2009. Oviposition, development and survivorship of the sweetpotato whitefly Bemisia tabaci on soybean, Glycine max, and the garden bean, Phaseolus vulgaris. Journal of Insect Science, vol. 9, pp. 1. http://dx.doi. org/10.1673/031.009.0101. PMid:19611218.

MOTA, M.V.S., DEMOLIN-LEITE, G.L., GUANABENS, P.F.S., TEIXEIRA, G.L., SOARES, M.A., SILVA, J.L., SAMPAIO, R.A. and ZANUNCIO, J.C., 2023. Chewing insects, pollinators, and predators on Acacia auriculiformis A. Cunn. ex Beth (Fabales: Fabaceae) plants fertilized with dehydrated sewage sludge. Brazilian Journal of Biology = Revista Brasileira de Biologia, vol. 83, pp. e248305. http://dx.doi.org/10.1590/1519-6984.248305. PMid:34669795.

NOVGORODOVA, T.A., 2015. Organization of honeydew collection by foragers of different species of ants (Hymenoptera: formicidae). European Journal of Entomology, vol. 112, no. 4, pp. 688-697. http://dx.doi.org/10.14411/eje.2015.077.

ÖBERG, S., MAYR, S. and DAUBER, J., 2008. Landscape effects on recolonisation patterns of spiders in arable fields. Agriculture, Ecosystems E'Environment, vol. 23, no. 1-3, pp. 211-218. http:// dx.doi.org/10.1016/j.agee.2007.06.005.

PETERSON, R.K., DAVIS, R.S., HIGLEY, L.G. and FERNANDES, O.A., 2009. Mortality risk in insects. Environmental Entomology, vol. 38, no. 1, pp. 2-10. http://dx.doi.org/10.1603/022.038.0102. PMid:19791592.

PIRES, E.M., SILVA, L.C., BATTIROLA, L.D., NOGUEIRA, R.M., BARRETO, M.R. and CORASSA, J. N., 2015. Triplaris americana L. (Polygonaceae), a new host plant for Aethalion reticulatum
(Linnaeus, 1767) (Hemiptera: Aethalionidae). Brazilian Archives of Biology and Technology, vol. 58, no. 1, pp. 31-33. https://doi. org/10.1590/S1516-8913201400039.

RAHMAN, M.M., RAHMAN, M.A., MIAH, M.G., SAHA, S.R., KARIM, M.A. and MOSTOFA, M.G., 2017. Mechanistic insight into salt tolerance of Acacia auriculiformis: the importance of ion selectivity, osmoprotection, tissue tolerance, and $\mathrm{Na}^{+}$ exclusion. Frontiers in Plant Science, vol. 8, pp. 155. http://dx.doi. org/10.3389/fpls.2017.00155. PMid:28421081.

RANA, V. and MAITI, S.K., 2018. Differential distribution of metals in tree tissues growing on reclaimed coal mine overburden dumps, Jharia coal field (India). Environmental Science and Pollution Research International, vol. 25, no. 10, pp. 9745-9758. http://dx.doi.org/10.1007/s11356-018-1254-5. PMid:29368202.

RODRÍGUEZ, J., CORDERO-RIVERA, A. and GONZÁLEZ, L., 2020. Characterizing arthropod communities and trophic diversity in areas invaded by Australian acacias. Arthropod-Plant Interactions, vol. 14, no. 4, pp. 531-545. http://dx.doi.org/10.1007/s11829020-09758-5.

SAGATA, K. and GIBB, H., 2016. The effect of temperature increases on an ant-Hemiptera-plant interaction. PLoS One, vol. 11, pp. e0155131. https://doi.org/10.1371/journal.pone.0155131.

SANCHEZ, A., 2015. Fidelity and promiscuity in an ant-plant mutualism: a case study of Triplaris and Pseudomyrmex. PLoS One, vol. 10, no. 12, pp. e0143535. http://dx.doi.org/10.1371/ journal.pone.0143535. PMid:26630384

SANCHEZ, J.A., LÓPEZ-GALLEGO, E. and LA-SPINA, M., 2020. The impact of ant mutualistic and antagonistic interactions on the population dynamics of sap-sucking hemipterans in pear orchards. Pest Management Science, vol. 76, no. 4, pp. 1422-1434. http://dx.doi.org/10.1002/ps.5655. PMid:31628776.

SILVA, J.L., DEMOLIN LEITE, G.L., DE SOUZA TAVARES, W., SOUZA SILVA, F.W., SAMPAIO, R.A., AZEVEDO, A.M., SERRÃO, J.E. and ZANUNCIO, J.C.. 2020. Diversity of arthropods on Acacia mangium (Fabaceae) and production of this plant with dehydrated sewage sludge in degraded area. Royal Society Open Science, vol. 7, no. 2, pp. 191196. http://dx.doi.org/10.1098/rsos.191196. PMid:32257306.

SISTEMA PARA ANÁLISES ESTATÍSTICAS E GENÉTICAS - SAEG, 2007 [accessed 30 June 2018]. Version 9.1 [online]. Available from: http://arquivo.ufv.br/saeg/

TONG, H., AO, Y., LI, Z., WANG, Y. and JIANG, M., 2019. Invasion biology of the cotton mealybug, Phenacoccus solenopsis Tinsley: current knowledge and future directions. Journal of Integrative Agriculture, vol. 18, no. 4, pp. 758-770. https://doi.org/10.1016/ S2095-3119(18)61972-0.

VENTURINO, E., ISAIA, M., BONA, F., CHATTERJEE, S. and BADINO, G., 2008. Biological controls of intensive agroecosystems: wanderer spiders in the Langa astigiana. Ecological Complexity, vol. 5, no. 2, pp. 157-164. http://dx.doi.org/10.1016/j.ecocom.2007.10.003.

WICKNESWARI, R. and NORWATI, M., 1993. Genetic diversity of natural-populations of Acacia auriculiformis. Australian Journal of Botany, vol. 41, no. 1, pp. 65-77. https://doi.org/10.1071/ BT9930065.

WONG, M.M., CANNON, C.H. and WICKNESWARI, R., 2011. Identification of lignin genes and regulatory sequences involved in secondary cell wall formation in Acacia auriculiformis and Acacia mangium via de novo transcriptome sequencing. BMC Genomics, vol. 12, pp. 342. http://dx.doi.org/10.1186/1471-216412-342. PMid:21729267.

ZANUNCIO, A.J.V., SERRÃO, J.E., PEREIRA, A.I.A., SOARES, M.A., WILCKEN, C.F., LEITE, G.L.D. and ZANUNCIO, J.C., 2015. Aethalion reticulatum (Hemiptera: Aethalionidae) feeding on Erythrina 
speciosa (Fabales: Fabaceae): first record of its host plant and damage characteristics. The Florida Entomologist, vol. 98, no. 1, pp. 175-177. http://dx.doi.org/10.1653/024.098.0130.

ZANUNCIO-JUNIOR, J.S., FORNAZIER, M.J., MARTINS, D.S., CHAMORRO-RENGIFO, J., QUEIRÓZ, R.B., LAZZARINI, A.L. and FERREIRA, P.S.F., 2017. Meroncidius intermedius (Orthoptera: Tettigoniidae): a threat to Brazilian banana. The Florida Entomologist, vol. 100, no. 3, pp. 669-671. http://dx.doi. org/10.1653/024.100.0329.
ZHANG, W., MCAUSLANE, H.J. and SCHUSTER, D.J., 2004. Repellency of ginger oil to Bemisia argentifolii (Homoptera: Aleyrodidae) on tomato. Journal of Economic Entomology, vol. 97, no. 4, pp. 13101318. http://dx.doi.org/10.1093/jee/97.4.1310. PMid:15384342.

ZOGRAFOU, K., ADAMIDIS, G.C., KOMNENOV, M., KATI, V., SOTIRAKOPOULOS, P., PITTA, E. and CHATZAKI, M., 2017. Diversity of spiders and orthopterans respond to intra-seasonal and spatial environmental changes. Journal of Insect Conservation, vol. 21, no. 3, pp. 531-543. http://dx.doi.org/10.1007/s10841-017-9993-z. 